Revisão das investigaçōes realizadas pela Fundação Oswaldo Cruz (FIOCRUZ) e Universidade Federal Rural do Rio de Janeiro (UFRRJ), com a colaboração da Superintendência de Campanhas de Saúde Pública do Ministério da

Sauide (SUCAM) e Secretaria Municipal de Saíde do Rio de Janeiro. Parcialmente subvencionado pelo Conselho Nacional de Desenvolvimento Cientifico e Tecnológico (CNPq).

* Fundação Oswaldo Cruz FIOCRUZ - RJ

** Superintendência de Campanhas de Saúde Pública do Ministério da Saide - SUCAM Rio de Janeiro.

*** Secretaria Municipal de Saúde do Rio de Janeiro.

*** Universidade Federal Rural do Rio de Janeiro - UFRRJ

\section{Leishmaniose visceral canina no Rio de Janeiro - Brasil}

\author{
Mauro Célio de A. Marzochi* \\ Sérgio Gomes Coutinho* \\ Paulo Chagastelles Sabroza* \\ Miguel Alves de Souza* \\ Pelágio Parigot de Souza** \\ Luciano Medeiros de Toledo*** \\ Francisco B. Rangel Filho****
}

No recente e mais meridional foco endêmico de leishmaniose visceral do Brasil, associado à presença de Lutzomyia longipalpis $e$ a infecção canina, os autores discutem a importância do cão doméstico como reservatório natural da infeç̧ão, assim como sumarizam os achados epidemiológicos, clínicos, parasitológicos e terapêtuticos da doença canina observados no Municipio do Rio de Janeiro, Brasil.

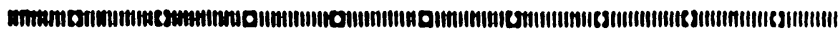

\section{INTRODUÇÃO}

A leishmaniose visceral (LV) canina, no Brasil, coexiste com a doenç a humana em todos os focos conhecidos sendo, porém, mais prevalente e, regra geral, precedendo à ocorrência de doença humana ${ }^{3}$.

Os cães infectados pela Leishmania donovani chagasi, à semelhança do calazar canino do Mediterrâneo, apresentam um bem conhecido espectro de características clínicas que podem variar de aparente estado sadio ao severo estágio final. Classicamente, na LV canina, tanto natural como experimentalmente induzida, se admite um período de incubação e prepatente de 3 a 6 meses até vários anos. Esta, invariavelmente, evolui para os estados latente ou patente que, por sua vez, em períodos variáveis de semanas, meses ou anos, podem evoluị para a forma aguda, subaguda, crônica ou regressiva ${ }^{3}, 9,21$. De modo geral, o quadro clínico se assemelha à doença humana, com febre irregular de longo curso, palidez de mucosas e um emagrecimento progressivo, até o estado de caquexia intensa, na fase terminal. A hiper- 
trofia do sistema fagocitário mononuclear (SFM), levando a esplenomegalia, hepatomegalia e adenopatia generalizada, é bastante freqüente, porém pouco referida. No entanto, os sinais mais evidentes estão relacionados às alterações cutâneas e de fâneros: perda de pelos, que é bastante freqüente, podendo ser focal ou generalizada; pequenas ulcerações crostosas, isoladas ou confluentes, observadas no focinho, orelhas e extremidades; descamação ou dermite furfuráceas, que acompanham a depilação; alongamento das unhas (grifose); opacificação da cómea (queratite intersticial), após conjuntivite purulenta; além de outros sinais como apatia, diarréia, hemorragia intestinal, paresia do trem posterior, edema e vômitos ${ }^{3}, 9,16,21$.

As alterações laboratoriais são semelhantes às que ocorrem no homem. A anemia do tipo normocrônico é freqüente $(62 \%$ dos casos), leucopenia moderada menos freqüente (33\%) e plaquetopenia mais rara, porém associada a fenômenos hemorrágicos ${ }^{14}$.

A eletroforese mostra uma disproteinemia com inversão das frações albumina e globulina, levando à hipergamaglobulinemia em $70,5 \%$ dos $\operatorname{casos}^{14}$, porém com acentuada elevação da fração beta ${ }^{3}$.

O diagnóstico de certeza se baseia na demonstração do parasito que é abundante nos tecidos do SFM e presente na pele, mesmo aparentemente sã. Na prática, a cultura em meio NNN do material obtido por punção de baço (difícil), fígado, medula óssea (tíbia) e linfonodos, principalmente 0 poplíteo, pelo fácil acesso, dão elevadas taxas de positivida$\mathrm{de}^{20}$.

Diversas reações imunológicas são utilizadas para a evidenciação de anticorpos específicos: reação de fixação de complemento, imunofluorescência indireta, imunodifusão, contra-imunoeletroforese e ELISA ${ }^{4}, 6,18,20,33,34$.

No Brasil, a reação de fixação de complemento $4.10,25,36$ $e$, mais recentemente, a imunofluorescência indireta ${ }^{8}$, realizadas em eluato de sangue dessecado em papel de filtro, têm sido utilizadas em inquéritos epidemiológicos extensos.

Paradoxalmente ao que ocorre na doença humana, o tratamento da LV canina pelos antimoniais tem sido considederado pouco eficiente ${ }^{3}, 14,15,16,21$.

Do ponto de vista epidemiológico, a doença canina é considerada mais importante que a doença humana, pois, além de ser mais prevalente, apresenta grande contingente de animais assintomáticos albergando parasitos no derma. Estes, assim como os cães doentes, representam melhor fonte de infecção para o inseto vetor, a Lutzomyia longipalpis, que o homem doente ${ }^{13}$. No entanto, esse mesmo fato possibilitaria a infecção intercanina, sem a participação do flebótomo, através de mordedura, durante brigas, do coito e, 
provavelmente também, pela ingestão de carrapatos que sugaram cães doentes ${ }^{3}$.

Além do homem e do cão, outros canídeos como as raposas Lycalopex vetulus, no Ceará ${ }^{12}$, e Cerdocyon thous, no Pará $^{19}$, são incriminadas como hospedeiros silvestres da LV.

\section{CARACTERISTICAS DAS ÁREAS ENDEMICAS NO MUNICÍPIO DO RIO DE JANEIRO}

Até o momento, as localidades do Município atingidas pela LV humana e canina se situam nas vertentes continentais norte (bairros de Realengo e Bangu), noroeste (Senador Camará) e oeste (Campo Grande) do Maciço da Pedra Branca, abrangendo a XVII e XVIII Regiões Administrativas.

Fig. 1 - Äreas de Leishmaniose visceral (LV) e de Leishmaniose tegumentar (LTA), nas encostas do Maciço da Pedra Branca - Município do Rio de Janeiro.

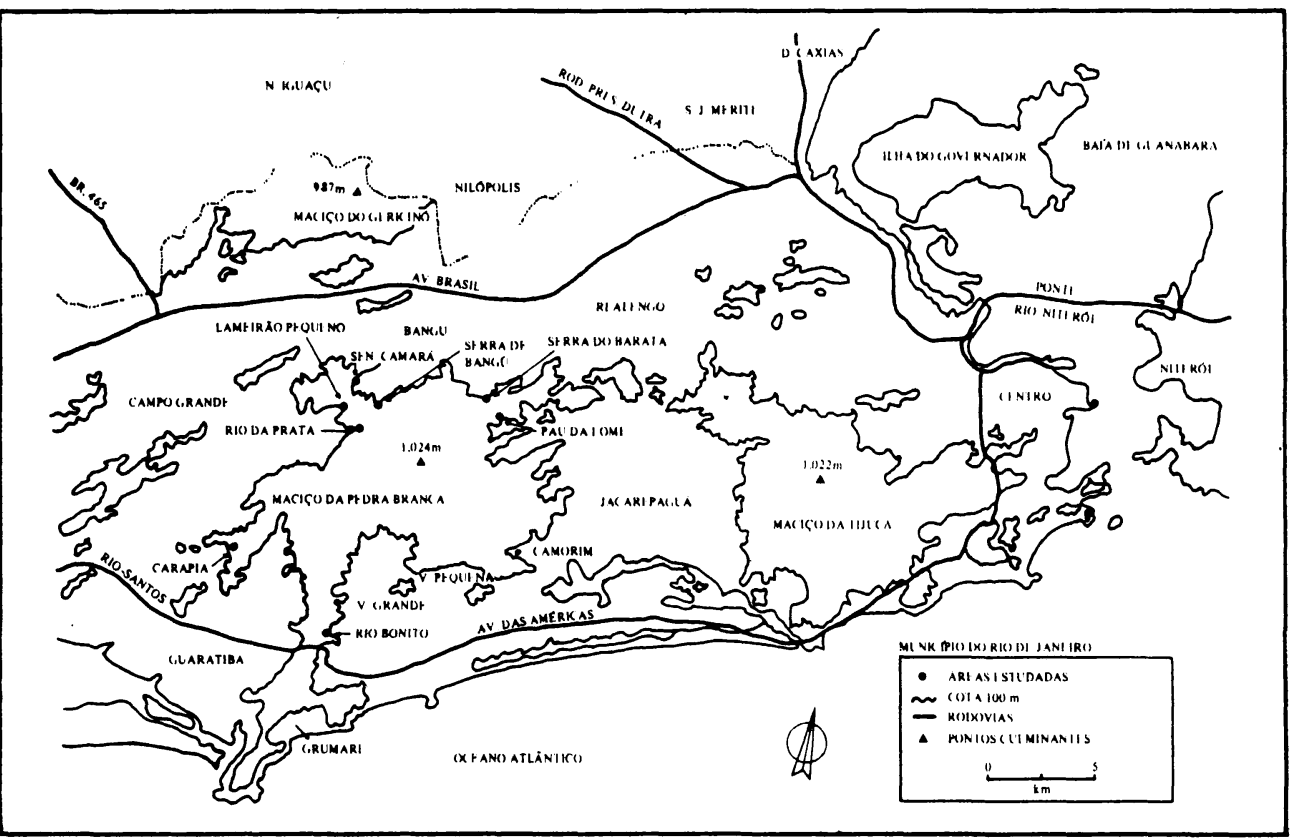

A vertente atlântica do mesmo maciço é voltada para o bairro de Jacarepaguá (XVI e XXVI Regióes Administrativas), onde a leishmaniose tegumentar é endêmica, com surtos esporádicos, e onde predomina a $\mathrm{Lu}$. intermedia ${ }^{41}$.

Este maciço, contraforte da Serra do Mar, situado no centro do Município, entre os paralelos $22^{\circ} 50^{\prime}$, latitude norte e $23^{\circ} 05^{\prime}$, latitude sul, e os meridianos $43^{\circ} 35^{\prime}$, leste e 
$43^{\circ} 20^{\prime}$, a oeste de Greenwich, é o conjunto montanhoso mais alto do município, formado por rochas graníticas e gnáissicas e cujo ponto culminante é o Pico da Pedra Branca (1024 m).

$\mathrm{O}$ clima predominante nas áreas de LV é do tipo AW (Koepen), com temperatura média anual de $27,7^{\circ} \mathrm{C}$. A precipitação pluviométrica total anual é maior que $1.400 \mathrm{~mm}$, com menos de $60 \mathrm{~mm}$ nos meses mais secos (julho e agosto), e a umidade relativa, pouco variável, em tomo de $75 \%^{39}$.

Estas são áreas periurbanas, com características rurais, geralmente de encostas de morros: a população predominante é de baixa renda, e as habitações são precárias e sem equipamentos sanitários ou iluminação élétrica, porém fixada à terra há muitos $\operatorname{anos}^{27}$ (Figura 1). Nessas áreas, tanto a doença de Chagas humana como a animal, assim como os triatomíneos transmissores não têm sido encontrados.

\section{INQUÉRITOS CANINOS REALIZADOS}

Em 1977, a partir da ocorrência de um caso humano de LV, na encosta da Serra de Bangu, vertente norte do maciço da Pedra Branca, periferia do bairro de mesmo nome, na cidade do Rio de Janeiro, da comprovação de sua autoctonicidade e da evidenciação da ocorrência de $97,9 \%$ de Lutzomyia longipalpis entre os flebótomos capturados nas cotas de altitude acima de 100 metros $^{41}$, foi realizado na mesma área, por pesquisadores da Fundação Oswaldo Cruz (FIOCRUZ), um inquérito canino.

Dos 30 cães existentes na localidade, examinaram-se 24 , nos quais se procedeu o exame clínico, coleta de fragmento de pele da orelha para impressão em lâmina e histopatologia e coleta de sangue em papel de filtro para sorologia.

Nestes, as aposições de fragmentos de pele em lâminas coradas foram negativas, e o material coletado para sorologia se perdeu. Mas, em um cão que se apresentava muito magro, com unhas discretamente crescidas e discretas ulcerações crostosas na extremidade das orelhas, evidenciaram. se formas amastigotas de Leishmania no corte histológico do fragmento de pele da orelha. O sacrifício e necrópsia desse animal, realizados após sua manutenção em cativeiro com alimentação adequada, por três meses, quando os sinais iniciais haviam desaparecido, não evidenciaram Leishmania em vísceras, linfonodos e medula óssea, mesmo após cultivo em NNN e inoculação em Hamster.

Após a ocorrência de novos casos humanos em 1979, na encosta do Morro do Barata, bairro de Realengo, vizinha à anterior, os pesquisadores da FIOCRUZ passaram a examinar apenas os cães clinicamente suspeitos. De cinco cães sus- 
peitos examinados e sacrificados no laboratório, em 2 deles evidenciou-se Leishmania em preparações coradas e em cultura de fragmentos de vísceras ${ }^{30}$.

Em 1980, o Ministério da Saúde (SUCAM) procedeu a um inquérito canino nas áreas de ocorrência de novos casos humanos (Realengo, Bangu e Senador Camará), através de coleta de fragmento de pele de orelha dos cães para exame parasitológico por oposição em lâmina e coleta de sangue em papel de filtro. As amostras de sangue dessecado foram enviadas à Universidade Federal de Minas Gerais, em Belo Horizonte, para a reação sorológica de fixação de complemento; dos 526 cães examinados pela SUCAM nessas áreas, evidenciaram-se $10(1,96 \%)$ cães soro-reagentes a $\mathrm{FC}$ e negativos ao exame de aposição e $10(1,96 \%)$ cães positivos ao exame direto e não-reagente a $\mathrm{FC}$; dos cães positivos a $\mathrm{FC}$, 5 foram recolhidos ao laboratório, sendo 1 procedente de Realengo, 1 de Bangu e 3 de Senador Camará, evidenciando-se em todos eles, mesmo com aspecto clínico normal, Leishmania donovani nos tecidos, tendo-se cultivado o parasito tanto de vísceras como de pele, aparentemente nor$\mathrm{mal}^{30}$.

Com a expansão da ocorrência de casos humanos para novas áreas periurbanas situadas na vertente oeste do mesmo maciço (bairro de Campo Grande), onde já havia sido referida a ocorrência de leishmaniose tegumentar americana $(\mathrm{LTA})^{23}$, a SUCAM realizou um inquérito canino mais amplo, após cadastramento da população humana, canina e das habitações, abrangendo todas as áreas onde foram referidos casos humanos de LV.

No período de junho a dezembro de 1982, procedeu-se a coleta de amostras de pele de orelha para aposição em lâmina e de sangue em papel de filtro para a pesquisa de anticorpos antileishmania pela reação de imunofluorescência indireta, na Fundação Oswaldo Cruz. Conforme Coutinho et a ${ }^{8}$, um total de 1342 cães foi examinado, evidenciando-se $4,3 \%$ de cães reagentes à IF nas áreas exclusivas de LV (Realengo, Bangu e Senador Camará), sendo de $12,7 \%$ a prevalência encontrada na área mista de Campo Grande e $8,6 \%$ de cães positivos em Pau da Fome (Jacarepaguá), vertente litorânea do maciço da Pedra Branca, onde têm ocorrido ex. clusivamente casos humanos e caninos de LTA.

Investigaçסes anteriores mostraram que a $L$. braziliensis braziliensis foi o único parasito isolado de casos humanos $\mathrm{e}$ caninos de LTA de Pau da Fome, onde o flebotomíneo mais freqüente e mais adaptado ao peridomicílio humano é a Lutzomyia intermedia ${ }^{31}$.

Como decorrência das medidas profiláticas, todos os cães suspeitos ou positivos aos exames parasitológicos ou sorológicos, nas áreas de LV, são sumariamente eliminados pela 
SUCAM ou recolhidos para estudo. No período de 1977 a 1983 , quarenta cães foram recolhidos e enviados ao laboratório para este fim*.

As idades desses cães variavam entre um e cinco anos, com predominância do sexo masculino. Dentre esses, 19 cães procediam de áreas de ocorrência exclusiva de LV (Realengo, Bangu e Senador Camará), observando-se sinais clínicos sugestivos da infeç̧ão em 37,8\% deles: emagrecimento - $100 \%$; linfadenopatia e depilação - $85,7 \%$; apatia - 71,4\%; ulcerações, descamação furfurácea e unhas alongadas - $42,9 \%$, além de outros sinais menos freqüentes, como evacuações sangüinolentas, ceratoconjuntivite e paresia do trem posterior $-14,3 \%$ (Figura 2 ).
"MARZOCHI, M.C.A.;

COUTINHO, S. G.; SOUZA,

W.J.S.; TOLEDO, L.M ; :

GRIMALDI FILHO, G.; MOMEN, H.; PACHECO, R.S.; SABROZA, $P C ;$ SOUZA, M.A.; RANGEL FILHO, F.B. \& TRAMONTANO, N.C. - 1985. Canine visceral leishmaniasis in Rio de Janeiro, Brazil. Clinical, parasitological, therapeutic and epidemiological findings (1977-1983). 1986.

Mem. Inst. Oswaldo Cruz $80(3)$ : 349.57, 1985.

Fig. 2 - Leishmaniose visceral canina no Município do Rio de Janeiro, A-D: sinais clínicos da LV em cães (emagrecimento, depilação, onicogrifose, esplenomegalia); E: cão Doberman antes da antimonioterapia; F: o mesmo cão após o tratamento.

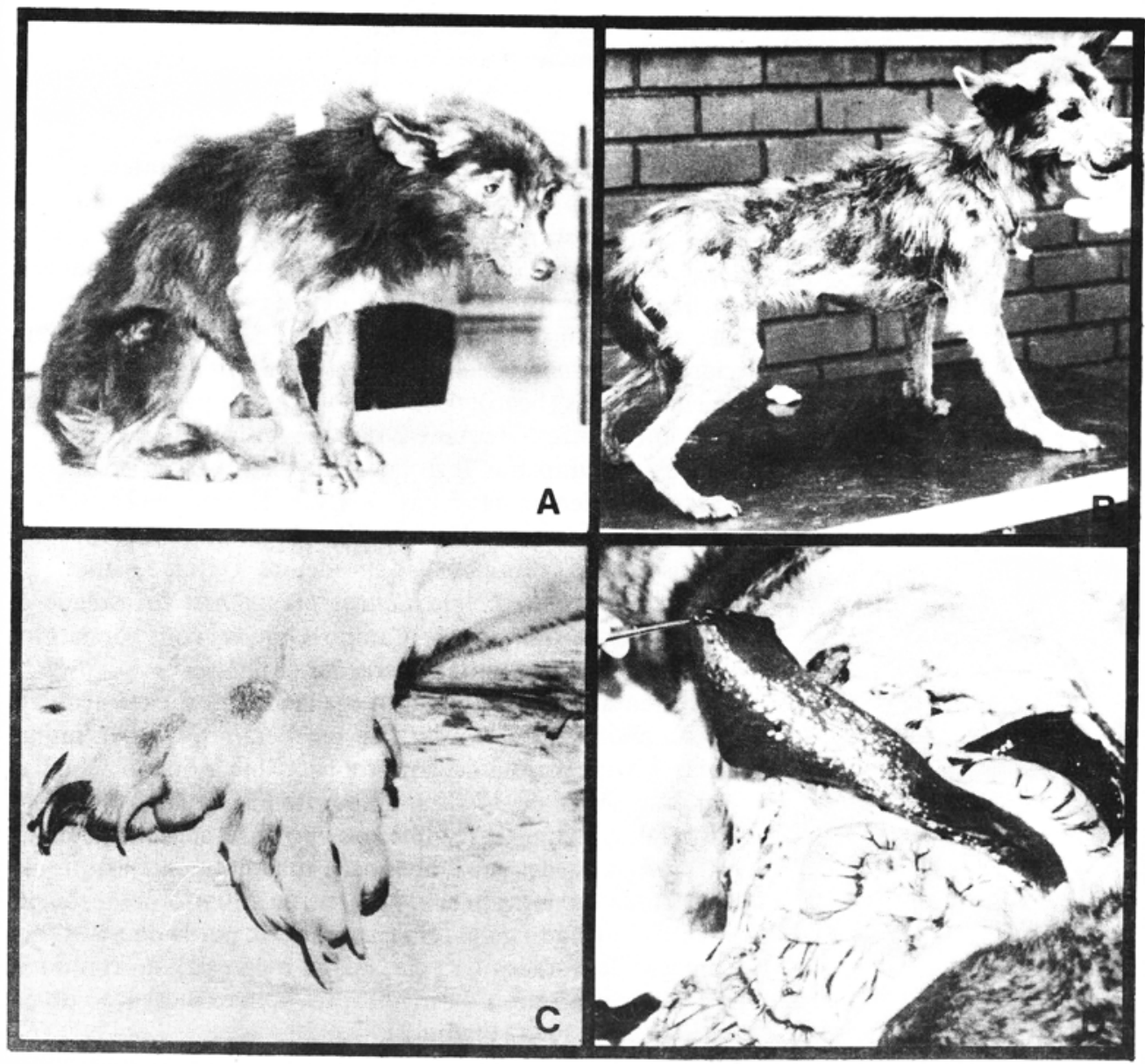

Caderno de. Saúde Pública, R.J., 1(4): 432-446, out/dez, 1985. 
Fig. 2 (continuação)

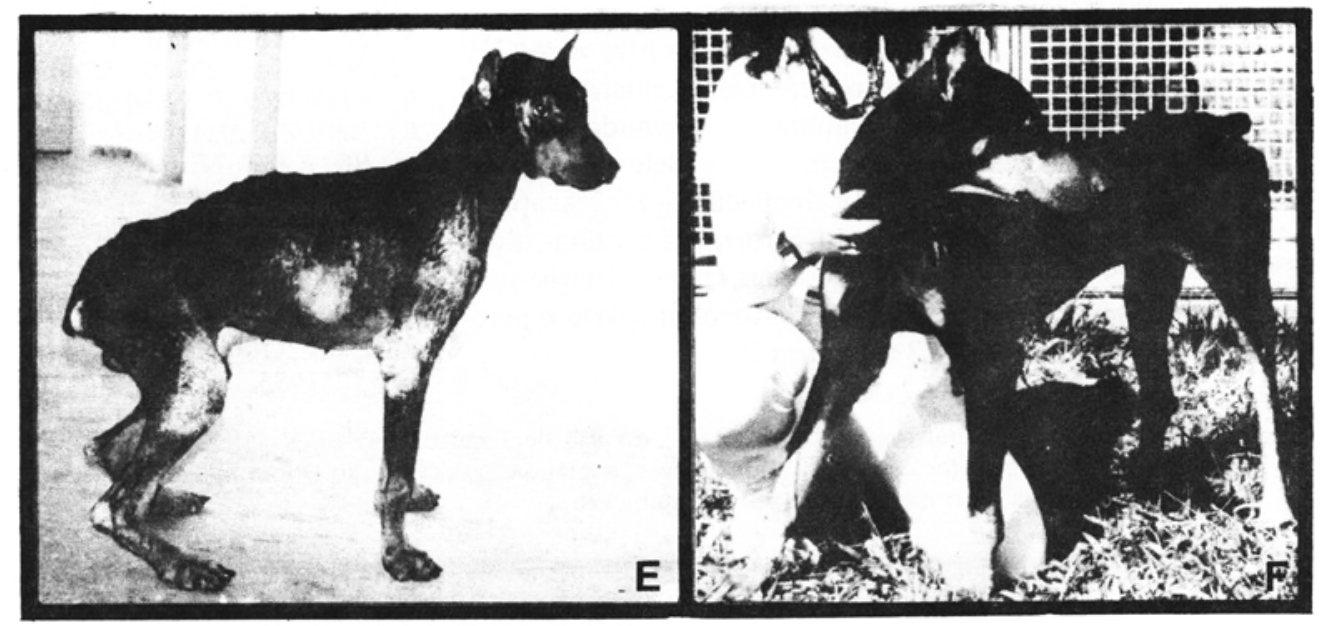

Outros 21 cães procediam de Campo Grande, onde tanto LV como LTA ocorrem. Nestes, os sinais de infecção pela Leishmania, principalmente ulcerações cutâneas e mucosas, foram observados em $76,2 \%$.

Tentativas de evidenciação ou isolamento do parasito realizadas nos 40 cães, através de biópsias de pele e mucosas, assim como de tecidos coletados após necrópsia, evidenciaram a presença de Leishmania em 39 , sendo $20 \%$ somente em vísceras (baço); $42,5 \%$ em vísceras e pele normal e em $35 \%$ em ulcerações cutâneas ou mucocu tâneas.

Das 11 amostras (estoques) de Leishmania isoladas de cães provenientes das áreas de LV e da área de LV + LTA, 10 foram caracterizadas como $L$. donovani, conforme seus esquisodemas, zimodemas e serodemas. $O$ único estoque caracterizado como $L$. braziliensis braziliensis foi isolado de linfonodo de um cão de Campo Grande, com parasitismo visceral e sem apresentar alterações cutâneas ${ }^{28}$.

A tentativa de tratamento realizada em 8 cães positivos para Leishmania donovani que receberam por via intramuscular $100 \mathrm{mg} / \mathrm{kg} /$ dia do antimonial (Glucantime - Rhodia), em duas séries de 17 dias, apresentou agravamento do estado geral, concomitante e mesmo após a terapêutica. Um dos cães tratados, de raça Doberman, aparentemente assintomático antes da terapêtica, apresentou intenso comprometimento do estado geral (emagrecimento, perda de pelos, descamação furfurácea da pele, apatia e paresia), morrendo 30 dias após o término da medicação, com exacerbação do parasitismo cutâneo (Figura 2). 


\section{COMENTÁRIOS}

O foco de leishmaniose visceral do Rio de Janeiro, com cerca de 50 casos humanos registrados até 1985 , parece ser o mais recente e o mais meridional do Brasil, associado à presença de Lutzomyia longipalpis e à infecção canina. Esta, tal como é observada em ou tras áreas endêmicas do País, parece ter prevalência significativamente mais elevada que a doença humana.

No entanto, tal como a doença humana, a leishmaniose canina parece também ser de implantação recente. Haja vista as dificuldades iniciais de encontro de animais infectados nos anos de 1977 a 1979 e sua relativa freqüência a partir de 1980, conforme revelam os inquéritos caninos, apesar das variações da metodologia empregada.

Por outro lado, as medidas de controle adotadas parecem ter influenciado significativamente a incidência da doença humana e canina ${ }^{29}$ (Tabela 1). Na área de Senador Camará, no entanto, onde se tem encontrado com freqüência a in-fecção canina, apenas um caso humano foi identificado.

\section{TABELA I}

Avaliação da prevalência de cães positivos a imunofluorescência indireta (IF $\geqslant 1: 40$ ) em áreas de leishmaniose visceral no Rio de Janeiro, durante as açōes de controle realizadas pela SUCAM.

\begin{tabular}{lcccrcrc}
\hline & \multicolumn{2}{c}{ Jul-Dez/82 (*) } & \multicolumn{3}{c}{ Jan-Dez/83 } \\
\cline { 2 - 7 } Áreas & $\begin{array}{c}\text { No de Cães } \\
\text { Examinados }\end{array}$ & $\begin{array}{c}\text { No de Cães } \\
\text { Positivos }\end{array}$ & $(\%)$ & $\begin{array}{c}\text { No de Cães } \\
\text { Examinados }\end{array}$ & $\begin{array}{c}\text { No de Cães } \\
\text { Positivos }\end{array}$ & $(\%)$ \\
\hline Realengo & 147 & 7 & $(4,8)$ & 354 & 8 & $(2,3)$ \\
Bangu & 169 & 6 & $(3,5)$ & 299 & 3 & $(1,0)$ \\
Senador Camará & 300 & 14 & $(4,7)$ & 489 & 9 & $(1,8)$ \\
Campo Grande & 353 & 45 & $(12,7)$ & 295 & 16 & $(5,4)$ \\
\hline Total & 969 & 72 & $(7,4)$ & 1.437 & 36 & $(2,5)$ \\
\hline
\end{tabular}

(*) Coutinho et al (1985)

Clinicamente, a infecção visceral canina observada no Rio de Janeiro não parece diferir da observada no nordeste do Brasil $^{3}, 5,11$, em Minas Gerais (Brener, 1957), assim como nas áreas mediterrâneas do sul da Europa e norte da África ${ }^{2}, 14,21,26,37$. No entanto, a proporção de cães infectados assintomáticos nas áreas exclusivas de LV $(63,2 \%)$ é similar à observada em Malta, $60 \%{ }^{2}$, na Grécia, $50 \%{ }^{26}$, na Toscânia (Itália), $59 \%{ }^{37}$, e mais baixa que a proporção encontrada por Alencar \& Cunha $^{5}$ no nordeste brasileiro, Ceará, $86 \%$. Cabe ressaltar, porém, que as características clínicas dos cães enviados ao nosso laboratório poderiam não re- 
presentar o universo dos cães infectados, estando, portanto, sujeito a vícios da triagem realizada pela SUCAM.

Por outro lado, enquanto na totalidade dos cães do Rio de Janeiro com sorologia (IF) positiva, 95\%, incluindo-se os assintomáticos, se demonstrou ou se isolou Leishmania ${ }^{8}$, na Toscania, Itália, Pozio et $\mathrm{al}^{37}$ encontraram o parasito em apenas $31,9 \%$ dos animais positivos a IF.

Lanotte et $\mathrm{al}^{21}$, em estudo realizado em cães com infecção natural, no sudeste da França (Cévennes), sugeriu a existência de dois grupos de formas clínicas, patentes e latentes, estando entre essas últimas as formas pré-clínicas (cerca de $90 \%$ ) e as formas resolutivas (cerca de 10\%).

Pozio et $\mathrm{al}^{37}$, em estudo longitudinal realizado na costa oeste da Itália, Toscânia, demonstraram que, após um ano, entre os cães inicialmente sintomáticos, $12 \%$ continuavam patentes e $88 \%$ morriam. Entre os assintomáticos, $52 \%$ apresentavam cura espontânea, com negativação da IF, $12 \%$ continuavam assintomáticos, $18 \%$ tomavam-se patentes e sintomáticos e $18 \%$ morriam no fim do período (Figura 3 ).

Fig. 3 - Tendência evolutiva da Leishmaniose visceral canina

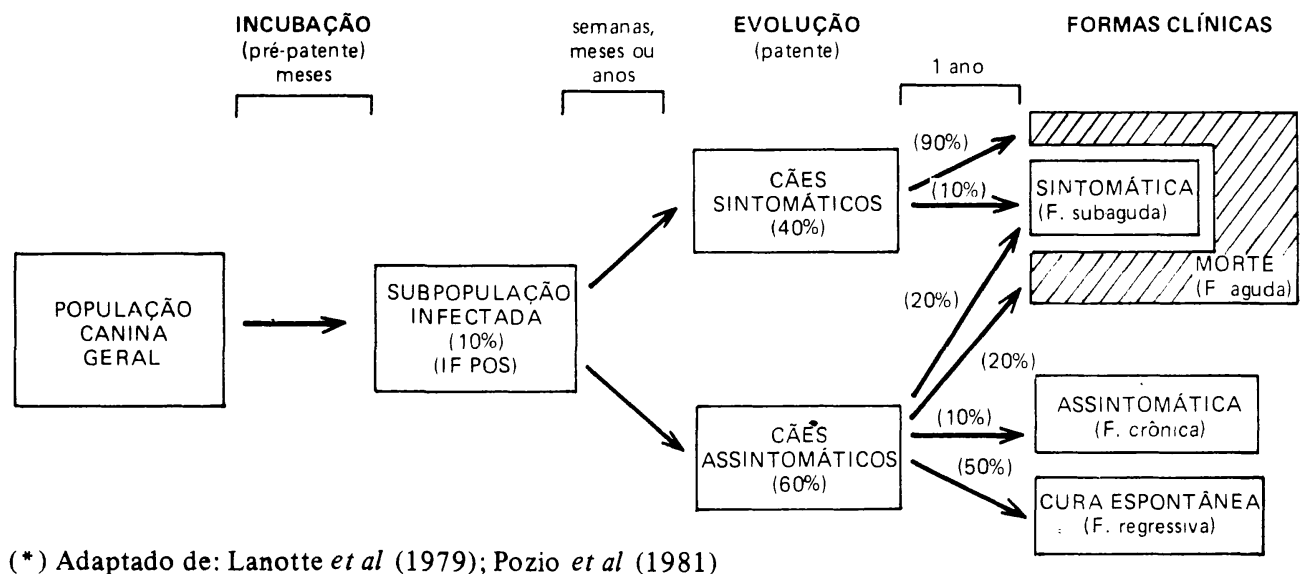

Em nossas observações anteriores, somente foi observado um caso de possível cura espontânea (forma resolutiva) no cão apreendido em 1977, uma vez que a maioria dos cães positivos eram sistematicamente eliminados no campo pela SUCAM ou sacrificados imediatamente em laboratório.

Segundo $\mathrm{Hommel}^{17}$, a perda de pelos tem sido explicada pela ação direta da Leishmania sobre o folículo piloso ou por um distúrbio do metabolismo do ácido pantotênico, decorrente de graves lesões hepáticas, ou ainda por deposição de imunocomplexos na membrana basal da pele, induzindo a um processo auto-imune que desencadeia a alopecia. 
$O$ alongamento anormal das unhas tem sido explicado pelo estímulo da matriz ungeal pelo próprio parasito ${ }^{22}$. No entanto, admitimos que a apatia do cão é grandemente responsável pelo não-desgaste natural das mesmas.

0 emagrecimento é atribuído à infiltração parasitária que comprometeria toda a mucosa do aparelho digestivo?, mas segundo Adler ${ }^{1}$, poderia ser explicado por um desequilíbrio protéico que levaria a importante albuminúria, podendo ser revertido pela ingestão de carne fresca. A explica. ção dos outros sinais como febre, eczema, ulceração de pele e mucosas, nódulos subcutâneos, queratite e fenômenos neurológicos é menos conclusiva ${ }^{17}$.

Os sinais clínicos observados entre nós, nas áreas de LV, foram relativamente constantes. $\mathrm{Na}$ área de Campo Grande, porém, onde a prevalência sorológica foi de $12,7 \%^{8}$, o número de cães com ulcerações foi proporcionalmente maior, tendo em quatorze deles se isolado ou demonstrado Leishmania apenas na lesão ulcerada e não em vísceras. A possibilidade de existência de áreas mistas (LV + LTA), no entanto, já havia sido admitida por Sherlock \& Almeida ${ }^{40}$ em localidades da Bahia.

No geral, não se observou nítida relação entre a presença e a intensidade dos sinais clínicos observados com o encontro de parasitas em víscera e/ou pele normal. Quanto à IF, os títulos mais elevados ocorreram nos cães que apresentaram comprometimento visceral.

A caracterização das 5 amostras de Leishmania donovani da área exclusiva de leishmaniose visceral e das 3 amostras da área de Campo Grande evidenciaram um padrão homogêneo entre as Leishmania isoladas dos cães e dos casos humanos de leishmaniose visceral ${ }^{24,32,35}$.

$\mathrm{Na}$ localidade de Lameirão Pequeno, área de Campo Grande, em um cão que apresentava ulceração típica de leishmaniose tegumentar na face interna da orelha, sem outros sinais de LV, a biópsia da lesão revelou crescimento de Leishmania, sendo caracterizada como L. donovani.

Este cão não pôde ser reestudado porque foi eliminado pela população local, preocupada com a ocorrência de um surto de LTA na localidade.

Por outro lado, na localidade de Rio da Prata (Campo Grande), em um cão com intenso parasitismo visceral, foi isolada em cultura Leishmania de um linfonodo que foi caracterizado como $L$. braziliensis braziliensis ${ }^{28}$.

$\mathrm{Na}$ área de Campo Grande, foi observada, com certa freqüência, a concomitância de ulcerações em mucosa (focinho), com ulcerações de pele (escroto, prepúcio ou outras regiōes).

A tentativa de tratamento de cães pelo antimonial, com dose acima da efetiva para a doença humana, não tem logra- 
do êxito, conforme o referido por diversos autores. Tal como foi por nós observado, Ranque \& Cabussu ${ }^{38}$, assim como Alencar ${ }^{3}$, descreveram o aparecimento de dermatite furfurácea em cães de pele previamente sadia, por ação do tratamento pelo antimonial, análoga, segundo os mesmos autores, à atribuída à reação de Herxheimer, e provavelmen. te devida, também, à excessiva liberação de material antigê. nico dos parasitos intracelulares mortos.

A evidenciação da ampla dispersão da enzootia canina, a partir de um foco inicial (Bangu) e da grande proporção de cães infectados assintomáticos, além da ineficácia do tratamento dos cães, comprova a importância dos inquéritos sorológicos e parasitológicos no diagnóstico epidemiológico, assim como na reavaliação das medidas de controles adota. das, dentre as quais inclui-se, obrigatoriamente, a eliminação de todos os cães positivos.

Essas medidas, no entanto, poderão sofrer grandes modificaçð̃es na vigência de um controle institucional da natalidade canina e/ou da utilização, nas áreas de risco, de uma vacina eficaz contra a leishmaniose canina. A possibilidade da aplicação dessa vacina poderia se constituir em eficiente meio de controle, tanto da doença canina como da doença humana. Tal procedimento, a nosso ver, seria tecnicamente possível, eticamente fácil de implantar e economicamente desejável, uma vez que, nas áreas endêmicas, poderia ser ministrado simultaneamente com a vacina anti-rábica, cujo programa de controle da raiva canina tem sido relativamen. te bem sucedido no País.

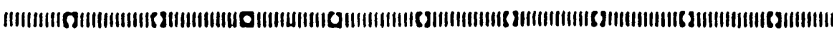

Based on the recent and most southernly endemic focus of visceral leishmaniasis in Brazil, associated with the presence of Lutzomyia longipalpis and canine infection, the authors discuss the importance of the household dog as a natural reservoir host of infection and also summarise the epidemiological, clinic, parasitological and therapeutical findings of the canine disease in the city of Rio de Janeiro, Brazil.

\section{REFERẼNCIAS BIBLIOGRÁFICAS}

1. ADLER, S. - Leishmania. In: Advances in Parasitology. New York, Academic Press, 1964. v. 2.

2. ADLER, S. \& THEODOR, O. - Mediterraneam Kala-azar. VII Further observations on canine visceral leishmaniasis. Proc. R. Soc., Ser. B., London, 116: 494-504, 1935. 
3. ALENCAR, J.E. - Calazar canino. Contribuição para o estudo da epidemiologia do calazar no Brasil. Fortaleza, Imp. Oficial, 1959. (Tese da Universidade Federal do Ceará).

4. ALENCAR, J.E.; ALMEIDA, Y.M.; SILVA, Z.F.; PAIVA, A.S. \& FONSECA, M.F. - Aspectos atuais do calazar no Ceará. R. bras. Malariol. D. trop., 26: 27-53, 1974.

5. ALENCAR, J.E. \& CUNHA, R.V. - Inquéritos sobre calazar canino no Ceará; novos resultados. $R$. bras. Malariol. $D$. trop., 15: 391-403, 1963.

6. BRENER, Z. - Calazar canino em Minas Gerais. Belo Horizonte, 1957. (Tese da Universidade Federal de Minas Gerais).

7. CATARSINI, O. - Epidemiologia e manifestazione cliniche della leishmaniosi del cane. $R$. Parasitol., 44: 83-7, 1981.

8. COUTINHO, S.G.; NUNES, M.P.; MARZOCHI, M.C.A. \& TRAMONTANO, N.C. - A survey for visceral and cutaneous leishmaniasis among 1342 dogs from areas in Rio de Janeiro (Brazil) where the human disease occurs. Mem. Inst. Oswaldo Cruz, 80:17-22, 1985.

9. CUNHA, A.M. - Infecções experimentais na leishmaniose visceral americana. Mem. Inst. Oswaldo Cruz, 33: 382-598, 1938.

10. CUNHA, R.V.; ALENCAR, J.E. \& ANDRADE, F.B. - Uso da reação de fixação de complemento para diagnóstico do Calazar canino em inquérito de massa. $R$. bras. Malariol. D. trop., 15: 405-10, 1963.

11. DEANE, L.M. - Leishmaniose visceral no Brasil; estudos sobre reservatórios e transmissores realizados no Estado do Ceará. Rio de Janeiro, Serviço Nacional de Educação Sanitária, 1956. (Tese da Universidade de São Paulo).

12. DEANE, M.P. \& DEANE, L.M. - Encontro de leishmanias nas vísceras e na pele de uma raposa em zona endêmica de calazar, nos arredores de Sobral, Ceará. O Hospital, 45: 419$21,1954$.

13. DEANE, L.M. \& DEANE, M.P. - Observações preliminares sobre a importância comparativa do homem, do cão e da raposa (Licalopex vetulus) como reservatório de Leishmania donovani em área endêmica de Calazar no Ceará. $O$ Hospital, 48: 61-70, 1955.

14. DEDET, J.P. - Les leishmanioses en Afrique du Nord. Bull. Inst. Pasteur, 77: 49-82, 1979.

15. Di LEO, R.; CASSANDRA, F.; MANISCALCO, N. \& NAPALI, G. - Indagine sulla leishmaniosi canina nel Comune di Sciacca. R. Parasitol., 42: 153-6, 1981.

16. DONATIEN, A. \& LESTOQUARD, F. - Remarques sur l'évolution de la leishmaniose générale du chien. Bull. Soc. Path. Exot., 31: 214-6, 1938.

Caderno de Saúde Pública, R.J., 1(4): 432-446, out/dez, 1985. 
17. HOMMEL, M. - The genus leishmania. Biology of the parasite and clinical aspects. Bull. Inst. Pasteur, Paris, 75: 5-102, 1978.

18. IVERSON, L.B.; CAMARGO, M.E.; VILLANOVA, A.; REICHMANN, M.L.A.B.; ANDRADE, E.A. \& TOLEZANO, J.E. - Inquérito sorológico para pesquisa de leishmaniose visceral em população canina-urbana do município de São Paulo - Brasil (1979-1982). R. Inst. Med. trop. São Pau10, $25: 310-7,1983$.

19. LAINSON, R.; SHAW, J.J. \& LUIS, Z.C. Leishmaniasis in Brazil. IV - The fox, Cerdocyon thous (L) as a reservoir of leishmania donovani, in Pará State. Brazil. Trans. R. Soc. trop. Med. Hyg., 63: 741-5, 1969.

20. LANOTTE, G.; RIOUX, J.A.; CROSET, H. \& VOLLHARDT, Y. - Ecologie des leishmanioses dans le sud de la France. 7. Dépitage de l'enzootic canine par les methodes immunosérologiques, Ann. Parasitol. Paris, 49: 41-62, 1974.

21. LANOTTE, G.; RIOUX, J.A.; PERIERS, J. \& VOLHARDT, Y. - Ecologie des leishmanioses dans le sud de la France. 10. Les formes évolutives de la leishmaniose viscérale canine. Elaboration d'une typologie bio-clinique à finalité épidemiologique. Ann. Parasit. Hum. Comp., 54: 277-95, 1979.

22. LESTOQUARD, F. \& DONATIEN, A. - Parasitisme de la matrice unguéale dans la leishmaniose génerale du chien. Bull. Soc. Pathol. Exot., 31:483-7, 1938.

23. LIMA, L.C.R.; MARZOCHI, M.C.A. \& SABROZA, P.C. - Flebotomíneos em área de ocorrência de leishmaniose tegumentar no bairro de Campo Grande, Rio de Janeiro, Brasil. R. bras. Malariol. D. trop., 33: 64-74, 1981.

24. LOPES, V.G.; MOMEN, H.; GRIMALDI, Jr., G.; MARZOCHI, M.C.A.; PACHECO, R.S. \& MOREL, C.M. - Schizodeme and zymodeme characterization of leishmania in the investigation of focil visceral and cutaneous leishmaniasis. $J$. Parasitol., 70: 89-98, 1984.

25. MAGALHÃES, P.A.; MAYRINK, W.; da COSTA, C.A.; MELO, N.M.; DIAS, M.; BATISTA, S.M.; MICHALICK' M.S.M. \& WILliAMS, P. - Calazar na zona do Rio Doce-Minas Gerais; Resultado das medidas profiláticas. $R$. Inst. Med. trop. São Paulo, 22:197-202, 1980.

26. MALAmOS, B. - Leishmaniasis in Greece. Trop. Dis. Bull., 44: $1-7,1947$.

27. MARZOCHI, M.C.A.; COUTINHO, S.G.; SOUZA, W.J.S. \& AMENDOEIRA, M.R.R. - Leishmaniose visceral - Calazar. J. bras. Med., 41: 69-84, 1981. 
28. MARZOCHI, M.C.A.; .GRIMALDI Jr., G.; MOMEN, H. \& McMAHON-PRATT, D. - Visceral Leishmania braziliensis braziliensis in a dog in Rio de Janeiro (BRAZIL). In: REUNIĀO ANUAL DE PESQUISA BÄSICA EM DOENÇA DE CHAGAS, 10. Caxambu, 1983. Anais. Caxambu, 1983. p. 3-75.

29. MARZOCHI, M.C.A.; SABROZA, P.C.; TOLEDO, L.M.; MARZOCHI, K.F.B.; TRAMONTANO, N.C. \& RANGEL, F. Leishmaniose visceral na cidade do Rio de Janeiro. Cad. Saúde públ., 1: 5-17, 1985.

30. MARZOCHI, M.C.A.; SOUZA, W.J.S; COUTINHO, S.G.; GRIMALDI Filho, \& MOMEN, H. - Leishmaniose visceral no Rio de Janeiro. I-Infecção canina em áreas de procedência de casos humanos autóctones. Cepas isoladas. In: CONGRESSO BRASILEIRO DE PARASITOLOGIA, 6. Belo Horizonte, 1981. Anais. Belo Horizonte, 1981. p. 73.

31. MARZOCHI, M.C.A.; SOUZA, W.J.S.; COUTINHO, S.G.; TOLEDO, L.M.; GRIMALDI Fo, G. \& MOMEN, H. - Evaluation of diagnostic criteria in human and canine mucocutaneous leishmaniasis in a Rio de Janeiro district where Leishmania braziliensis braziliensis occurs. In: REUNIÃO ANUAL DE PESQUISA BÄSICA EM DOENÇA DE CHAGAS. 9. Caxambu, 1982. Anais. Caxambu, 1982. p. 63.

32. MOMEN, H.; GRIMALDI Jr., G. \& MARZOCHI, M C.A. Identification of New World Leishmania isolates by agarose gel electrophoresis and polyacrylamide gel isoelectrofocusing. J. Cell. Biochem., 7A, 70:29, 1983.

33. MONSUETO, S.; MICELI, M.D. \& QUARTARARO, P. Counterimuno-electrophoresis (CEIP) and ELISA test in the diagnosis of canine leishmaniasis. Ann. trop. Med. Parasitol., 76: 229-31, 1982.

34. NUSSENZWEIG, U.; NUSSENZWEIG, R.G. \& ALENCAR J.E. - Leishmaniose visceral canina; reação de fixação de complemento com antígeno extraído do bacilo da tuberculose. O Hospital, 51: 325-32, 1957.

35. PACHECO, R.S.; MOMEN, H.; GRIMALDI Jr., G.; MARZOCHI, M.C.A.; MOREL, C.M. \& BADARÓ, R. - A comparative biochemical analysis of isolates of visceral leishmaniasis from the states of Bahia and Rio de Janeiro. In: JAPAN-BRAZIL SIMPOSIUM ON SCIENCE AND TECHNOLOGY, 4. São Paulo, 1984. Proceedings. São Paulo, $\mathrm{CNPq} /$ Academia de Ciências do Estado de São Paulo, 1984. V. 2 p. 58-83.

36. PELLEGRINO, J. \& BRENER, Z. - Reação de fixação de complemento com sangue dessecado no diagnóstico do calazar canino. R. bras. Malariol. D. trop., 10:39-44, 1958.

37. POZIO, E.; GRANDONI, L.; BETTINI, S. \& GRANICIA, M. Leishmaniasis in Tuscany (Italy): VI Canine Leishmaniasis in the focus of Monte Argentario (Grosseto). Acta Troppica, 38: 383-93, 1981.

Caderno de Saúde Pública, R.J., 1(4) : 432-446, out/dez, 1985. 
38. RANQUE, J.M. \& CABASSU, H. - Le diagnostic précoce de la leishmaniose canine par la ponction ganglionaire; réfléxions à propos de soixante examens positifs obtenus en dix mois dans la région marseillaise. Bull. Ac. Nat. Med, $132: 339-41,1948$.

39. RIO DE JANEIRO (cidade). Secretaria Municipal de Planejamento e Coordenação Geral. Informações básicas da cidade do Rio de Janeiro. Rio de Janeiro, 1979.

40. SHERLOCK, I.A., \& ALMEIDA, S.P. - Notas sobre leishmaniose canina no estado da Bahia. $R$. bras. Malariol. $D$. trop., 22: 231-42, 1970.

41. SOUZA, M.A.; SABROZA, P.C.; MARZOCHI, M.C.A.; COUTINHO, S.G. \& SOUZA, W.J.S. - Leishmaniose visceral no Rio de Janeiro. 1. Flebotomíneos da área de procedência de caso humano au tóctone. Mem. Inst. Oswaldo Cruz, 76: $161-8,1981$ 